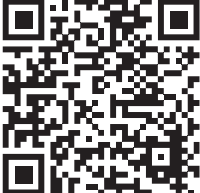

* Licenciatura en Enfermería, Facultad de Estudios Superiores Zaragoza, Universidad Nacional Autónoma de México. México.

Correspondencia: IES, ivonne1290@hotmail.com Conflicto de intereses: Ninguno.

Citar como: EspinozaSánchez I, de la PeñaLeón B, Luna-Pérez D, Lezana-Fernández MÁ, Meneses-González F. Manejo de enfermería en complicaciones de fístula arteriovenosa para hemodiálisis. Rev CONAMED. 2021; 26(1): 48-52. https://dx.doi. org/10.35366/99128 Financiamiento: Este artículo fue financiado con recursos y ayuda de la CONAMED.

Recibido: 12/01/2021.

Aceptado: 16/03/2021.

\section{Manejo de enfermería en complicaciones de fístula arteriovenosa para hemodiálisis}

\author{
Nursing management in complications of \\ arteriovenous fistula for hemodialysis \\ Ivonne Espinoza-Sánchez, Belinda de la Peña-León,* David Luna-Pérez, \\ Miguel Ángel Lezana-Fernández, Fernando Meneses-González*
}

\section{RESUMEN}

Introducción: La enfermedad renal crónica es un problema de salud pública. La hemodiálisis es uno de los principales tratamientos utilizados para tratar esta enfermedad que se encuentra en su etapa terminal. Esta intervención requiere de un acceso vascular seguro y al inicio de las sesiones de hemodiálisis el personal de enfermería debe realizar cuidados y seguir protocolos exhaustivos para la supervivencia y permeabilidad del acceso vascular. Caso clínico: Se trata de un enfermo renal crónico con tratamiento de hemodiálisis que presentó pérdida de funcionalidad de la fístula arteriovenosa para su tratamiento. Conclusión: El manejo de las fístulas arteriovenosas en una hemodiálisis resalta la importancia de conocer el procedimiento correcto respecto al uso de accesos vasculares por parte del personal de enfermería, así como del autocuidado por parte del paciente.

Palabras clave: Hemodiálisis, fístula arteriovenosa, acceso vascular.

\begin{abstract}
Introduction: Chronic kidney disease has been recognized as a global public health problem. Hemodialysis one is one of the main treatments used to combat this disease which is in its terminal stage. This intervention requires which you need a safe vascular access to be able to carry out the procedure correctly. Clinical case: This is a male patient diagnosed with chronic kidney disease or undergoing hemodialysis treatment who presented loss of functionality of the arteriovenous fistula for hemodialysis treatment. Conclusion: The management of arteriovenous fistulas in hemodialysis underline the importance of knowing the correct procedure regarding the use of vascular access for hemodialysis by the nursing staff as well as self-care by the patient.
\end{abstract}

Keywords: Hemodialysis, arteriovenous fistula, vascular access.

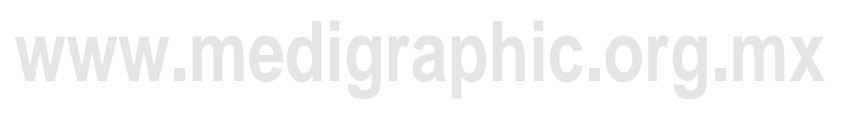




\section{INTRODUCCIÓN}

La enfermedad renal crónica se reconoce como un problema de salud pública, se ha estimado que, en la actualidad, alrededor de 6.2 millones de mexicanos con diabetes tienen insuficiencia renal en sus distintas etapas, sin que necesariamente todos ellos sepan que la padecen. La hemodiálisis es uno de los tratamientos sustitutivos que se utilizan para la enfermedad renal crónica terminal, es un procedimiento invasivo que permite extraer los productos tóxicos generados por el organismo que se han acumulado en la sangre a través de una máquina y filtros especiales de diálisis, que no está exento de producir complicaciones. En la aparición de complicaciones intervienen factores de riesgo como hipervolemia, uremia, hipertensión arterial y anemia.?

Para la realización de la hemodiálisis se requiere de un acceso vascular. Los accesos vasculares pueden ser accesos arteriovenosos: fístulas, prótesis o injertos arteriovenosos y los catéteres venosos centrales.

La fístula arteriovenosa interna es el acceso vascular más seguro y de mayor duración, se busca fortalecer las paredes de la vena para que esté en condiciones de recibir las agujas empleadas en la hemodiálisis. ${ }^{2}$ Al inicio de las sesiones de hemodiálisis se debe tener cuidados exhaustivos y protocolizados del acceso vascular. El personal de enfermería mediante el cuidado y el entrenamiento del paciente en el autocuidado de la fístula arteriovenosa contribuye directamente a la permeabilidad y supervivencia del acceso vascular. ${ }^{3}$

\section{PRESENTACIÓN DEL CASO}

Masculino de 27 años con diagnóstico de enfermedad renal crónica diagnosticada en agosto de 2009, secundaria a glomerulonefritis además de cursar con hipertensión arterial sistémica y síndrome anémico. Inició tratamiento sustitutivo con diálisis peritoneal continua ambulatoria en octubre de 2009 y diálisis peritoneal automatizada en diciembre del mismo año. Debido a mala evolución por contar con un peritoneo de baja efectividad para la diálisis, se revaloró un cambio de tratamiento y se sustituyó por hemodiálisis. El tratamiento inició en enero de 2010 y se le instaló catéter venoso tipo Mahurkar en la vena subclavia derecha. El programa de hemodiálisis se aplicaba en sesiones semanales.

El paciente no tenía posibilidades de un trasplante renal por donador vivo, por lo que en junio de 2011 le fue colocada una fístula interna por el Servicio de Angiología y las sesiones de tratamiento pasaron a ser tres por semana.

En mayo de 2013 acudió a sesión de hemodiálisis con enfermera en turno, quien se encargaba de brindar las sesiones. En hoja de evolución se reportó hipotensión y se estabilizó. Sin embargo, al término de la sesión el paciente presentó nuevamente hipotensión y se le administró solución glucosada al 50\%. Se dio por terminada la sesión, desconectándolo del riñón artificial sin complicaciones y de acuerdo al procedimiento registrado en hoja de enfermería.

El día 18 de mayo el paciente refirió que observó edema en el brazo donde se encontraba el acceso vascular debido a la última sesión que se le otorgó, además de percatarse de la ausencia de thrill. El thrill es una vibración palpable de la fístula arteriovenosa que se debe al flujo de sangre que circula. ${ }^{3}$ En hojas de enfermería se indica que en caso de presentar alguna anomalía en su acceso vascular debe acudir de inmediato a urgencias para prevenir complicaciones; sin embargo, el paciente no acudió hasta el día 20 de mayo, en su nueva sesión de hemodiálisis con la misma enfermera, quien, antes de realizar punción, hizo observación, palpación y auscultación exhaustiva en el acceso para verificar la ausencia de thrill, posteriormente se realizó una punción en fístula sin obtener resultado, por lo que se confirmó la pérdida de la funcionalidad de la fístula. Fue valorado por personal médico y se indicó la colocación de un catéter tipo Mahurkar, pero debido a complicaciones para obtener el acceso vascular se decidió valorar por Servicio de Cirugía Vascular y se le realizó un acceso vascular temporal con un catéter Permacath, a partir de allí su tratamiento continuó.

\section{DISCUSIÓN}

El acceso vascular se ha utilizado para realizar la hemodiálisis en pacientes con enfermedad renal crónica terminal. Este acceso por sí solo condiciona al éxito o al fracaso del tratamiento. 
Existen diferentes complicaciones por no tener los cuidados suficientes para mantenerlo permeable.

Los principales indicadores que se deben tomar en cuenta para poder realizar este tipo de procedimientos se basan en contar con profesionales certificados que cuenten con los conocimientos eficientes, además de que la unidad cuente con el equipamiento necesario para su realización óptima. ${ }^{4}$ Los cuidados que debe realizar el profesional de enfermería en cada sesión de hemodiálisis son realizar las medidas de precaución universal como el uso de cubrebocas, guantes y campos estériles durante la manipulación del catéter y en su conexión y desconexión con la máquina, realizar un examen exhaustivo de la fístula mediante su observación directa, palpación y auscultación. No se debe realizar la punción sin comprobar antes su funcionamiento, así como conocer el tipo de acceso, su anatomía y la dirección del flujo sanguíneo para programar las zonas de punción.

Asimismo, se debe valorar de manera objetiva el estado físico del paciente, si presenta síntomas como son disnea, edema o debilidad, así como de forma subjetiva en el que el paciente mencione presentar algún tipo de anomalía para brindar el tratamiento adecuado y corregirlos de manera oportuna antes de cada sesión. Por otra parte, los parámetros de la máquina de hemodiálisis deben mantenerse íntegros mientras se esté llevando a cabo el procedimiento, se debe verificar que los elementos que la componen no presenten fugas o roturas, además de mantener las conexiones visibles y corroborar que no haya objetos ajenos que puedan causar accidentes. Antes del inicio de la sesión se debe cerciorar de que el paciente no tenga indicación de estudios de gabinete o administración de medicamentos. Mantener registros clínicos de los signos vitales del paciente, así como reacciones adversas que se presenten se deben registrar en hojas de valoración que estén dentro de su expediente clínico. ${ }^{5}$

De acuerdo con el algoritmo de la «Guía de práctica clínica de manejo de acceso vascular en hemodiálisis», las acciones de enfermería para la punción de la fístula deben ser las presentadas en la Figura $7 .{ }^{6}$

Cuando la sesión de hemodiálisis termina se deben retirar las agujas y ejercer una compresión leve y no circular con gasas para lograr hemostasia, este cuidado es crucial para evitar la formación de hematomas después de las sesiones. Entre las principales causas de disfunción de la fístula son la trombosis y estenosis. La estenosis está producida generalmente por punciones repetidas sobre la misma zona. Se identifica por la aparición

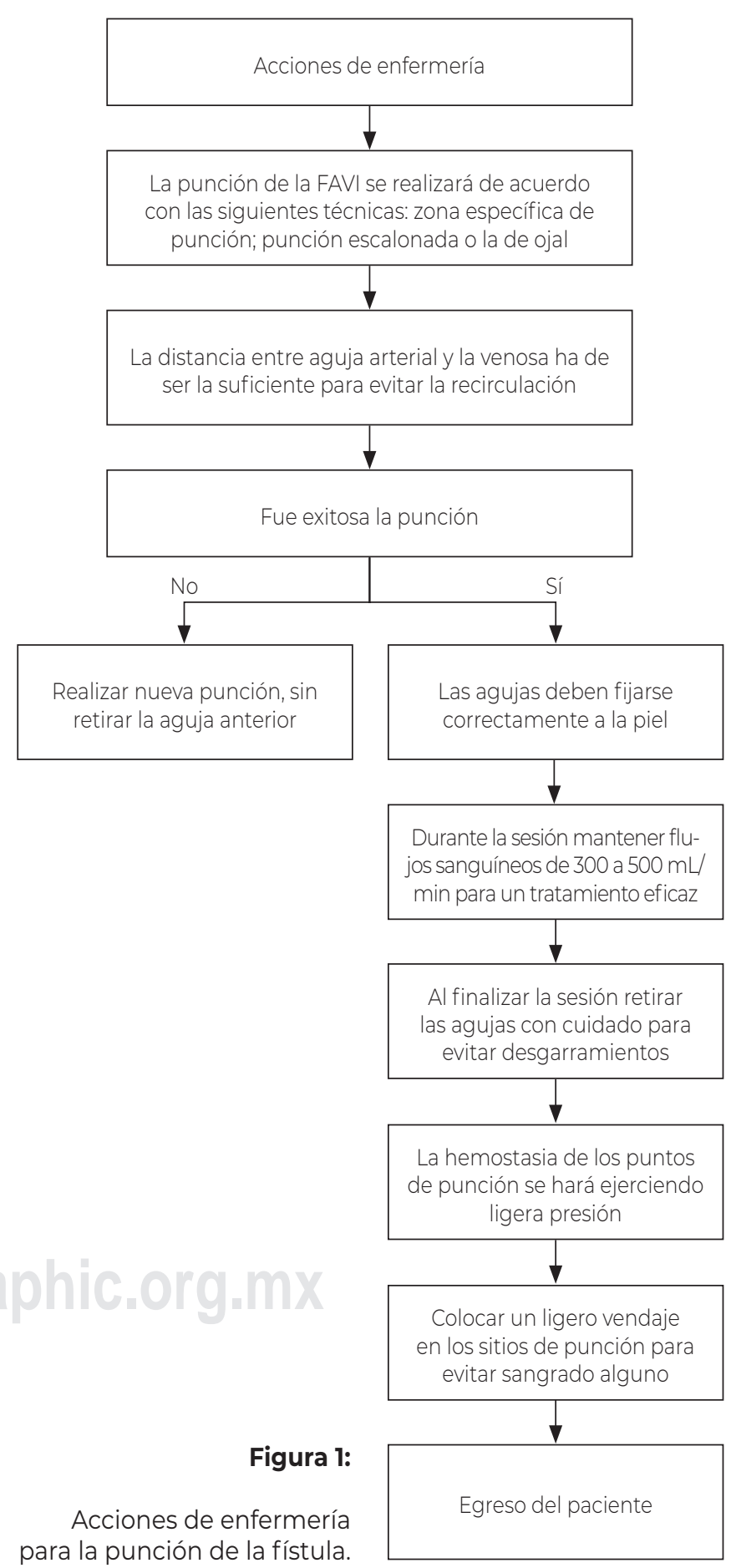


de dilatación y adelgazamiento de las paredes. La fístula arteriovenosa debe presentar un thrill palpable y audible con el estetoscopio, el tono debe ser claro y potente. En caso contrario, es indicativo de probable estenosis. Si se percibe esta disfunción se deben iniciar exploraciones para confirmarla y resolver el problema antes de que se produzca una trombosis. En la mayoría de los casos de trombosis, la causa una estenosis en la parte más proximal de la anastomosis en la fístula arteriovenosa. ${ }^{6-8}$ La trombosis de la fístula arteriovenosa es una complicación importante que puede resultar en la pérdida de la fístula para la hemodiálisis.

De acuerdo con un estudio realizado en el Hospital General de México «Dr. Eduardo Liceaga»la principal causa de pérdida de la fístula es debido a una trombosis (51\%) y esto puede deberse a la detección tardía de complicaciones. ${ }^{9}$

En el presente caso hubo pérdida de la funcionalidad de la fístula arteriovenosa. Sin embargo, se cuentan con registros clínicos que reportan valoraciones previas de las sesiones de hemodiálisis conducidas al paciente sin complicaciones. También existen reportes posteriores al acontecimiento en los cuales se menciona que se siguen los protocolos de la técnica correcta; además, existe evidencia de que el procedimiento fue realizado por personal certificado y se tienen registros de informes al paciente en los que se indican que al momento de presentar alguna anomalía, y de ser necesario, debe acudir a urgencias médicas inmediatamente, este último hizo caso omiso a dicha indicación y esperó la próxima sesión.

El autocuidado por parte del paciente es crucial para poder mantener la supervivencia de la fístula. La clasificación de intervenciones de enfermería (Nursing Interventions Classification) incluye el mantenimiento del acceso para diálisis, la cual es definida como la conservación de lugares de acceso vascular. A través de esta intervención la enfermera puede planificar los cuidados más adecuados para educar al paciente en los autocuidados de su fístula. Entre las actividades imprescindibles que se encuentran es que el paciente compruebe si en el lugar de acceso existe eritema, edema, hemorragia, hematoma y disminución de la sensibilidad, comprobar la funcionalidad de la fístula a intervalos frecuentes, evitar la compresión mecánica del lugar, así como evitar venopunción y toma de presión arterial. ${ }^{10}$

\section{CONCLUSIONES}

El acceso vascular es un elemento fundamental para poder realizar la hemodiálisis y es de vital importancia que los profesionales de la salud de las diferentes especialidades que participan en el tratamiento de este tipo de enfermedad conozcan correctamente la forma de realizar un procedimiento de alta calidad para mejores resultados. Un adecuado cuidado en el acceso vascular es primordial para contribuir en el tratamiento de la enfermedad renal crónica terminal para el paciente, ya que ayuda a mejorar su estado de salud tanto físico como emocional, si bien no es un tratamiento curativo, ayuda a preservar aún más la vida del individuo.

Por otra parte, es importante que el personal que realice este tipo de procedimientos se mantenga apegado a las guías de práctica clínica en el manejo de accesos vasculares en hemodiálisis, así como a la Norma Oficial Mexicana NOM-003SSA3-2010 para la práctica de la hemodiálisis, ya que son documentos que se fundamentan en un manejo adecuado del tratamiento. Además, el personal de enfermería debe sustentarse de los cuidados que brinda al paciente guiándose de las taxonomías en enfermería como el NIC (Nursing Interventions Classification) y de los documentos oficiales antes mencionados. Por último, es necesario brindarle información al paciente respecto a los autocuidados que debe realizar para mantener un mejor control de su enfermedad, por lo que todo debe ser reconocido como una serie de procedimientos que realizan en conjunto el personal de salud con el paciente.

\section{REFERENCIAS}

1. Tamayo y Orozco JA, Lastiri Quirós SH. La enfermedad renal crónica en México. Hacia una política nacional para enfrentarla. México: Academia Nacional de Medicina de México; 2016. Disponible en: https://www. anmm.org.mx/publicaciones/ultimas_publicaciones/ ENF-RENAL.pdf

2. Jiménez Almonacid P. Fístulas arteriovenosas para hemodiálisis. En: Lorenzo-Sellarés V, López-Cómez JM, editores. Nefrología al día [Internet]. Barcelona: Sociedad Española de Nefrología; 2015. pp. 401-410 Disponible en: https://www.nefrologiaaldia.org/es-articulo-fistulasarteriovenosas-hemodialisis-38. 
3. Fernández Medina IM. Cuidados de la fístula arteriovenosa durante la sesión de hemodiálisis. ENE. Revista de Enfermería. 2013; 7 (1).

4. Rodríguez Hernández JA, González Parra E, Gutiérrez Julián JM, Segarra Medrano A, Almirante B, Martínez MT et al. Cuidados del acceso vascular. guías de acceso vascular en hemodiálisis. Nefrología. 2005; 25 (1): 29-33. Disponible en: https://www.revistanefrologia.com/espdf-X0211699505030797

5. Andrade Nívea C. Fistulas arteriovenosas del cuidado de enfermería: revisión de la literatura. Revista Científica de Conocimiento Multidisciplinar. 2016; (9): 8-106.

6. Accesos vasculares en hemodiálisis. En adultos con insuficiencia renal crónica en segundo y tercer nivel de atención. Guía de Práctica Clínica. México CENETEC; 2013.
7. Barba Velez A, Ocharan Corcuera J. Accesos vasculares para hemodiálisis. Gac Med Bilbao. 2011; 108 (3): 63-68.

8. Secretaría de salud. Norma Oficial Mexicana NOM-003SSA3-2010, Para la práctica de la hemodiálisis. [Fecha de acceso: 15 de enero del 2020] Disponible en: http://www. dof.gob.mx/normasOficiales/4093/Salud/Salud.htm.

9. Casares-Bran T, Olivares-Cruz S, Lecuona-Huet N, FabiánMijangos W, Rodríguez-López E, Betanco-Peña A et al. Fístulas arteriovenosas para hemodiálisis: tres años de experiencia en el Servicio de Angiología del Hospital General de México "Dr. Eduardo Liceaga". Rev Mex Angiol. 2017; 45 (4): 163-169.

10. Butcher HK, Bulecheck GM, Dochterman JM, Wagner CM. Clasificación de intervenciones de enfermería (NIC). 7a edición. España: Elsevier; 2019. 